\title{
B/P Doping in Application of Silicon Oxynitride Based Integrated Optics
}

\author{
F. Sun'1, M. G. Hussein ${ }^{2}$, K. Wörhoff' ${ }^{1}$, G. Sengo ${ }^{1}$ and A. Driessen ${ }^{1}$ \\ 1. University of Twente, Postbus 217, 7500AE Enschede, The Netherlands \\ 2. OTB Display, P.O. Box 7005, 5605 JA Eindhoven, The Netherlands
}

Silicon oxynitride ( $\mathrm{SiON})$ is a highly transparent amorphous material over a wide range of wavelengths, and has won excellent reputations in its low loss, design flexibility and high thermal stability, which has made it a very competent candidate material for integrated optics[1-2]. Growth of SiON layer can be realized by CMOScompatible chemical vapor deposition (CVD) technologies[3]. However, due to the introduction of hydrogencontaining precursors, such as ammonia $\left(\mathrm{NH}_{3}\right)$, silane $\left(\mathrm{SiH}_{4}\right)$ and dichlorsilane $\left(\mathrm{SiH}_{2} \mathrm{Cl}_{2}\right)$, as-deposited $\mathrm{SiON}$ layers may show hydrogen-related loss properties, especially in wavelength window for telecommunication application[4]. With the help of high temperature annealing, the influence of hydrogen can be eliminated. Furthermore, treatment under high temperature can also help to improve the reflow property of SiON channel waveguides and/or their upper claddings, which will accordingly result in further reduction of propagation loss due to the smaller sidewall roughness and better coverage of cladding layers. Unfortunately, the necessary temperatures of annealing and reflow for pure $\mathrm{SiON}$ are too high to be tolerable for most integrated electronic devices, which is a big challenge in the monolithic integration of electronics and photonics.

In this paper, gaseous precursors containing boron or phosphorous were intentionally introduced in the deposition of $\mathrm{SiON}$ layers and upper $\mathrm{SiO}_{2}$ claddings. The measurements show that the as-deposited $\mathrm{B} / \mathrm{P}$-doped SiON layers contain less hydrogen than undoped layers, as can be seen in Fig. 1. Furthermore, the necessary annealing temperature for elimination of hydrogen related absorption (propagation loss) is greatly reduced in B/P-doped layers, also shown in Fig. 1.

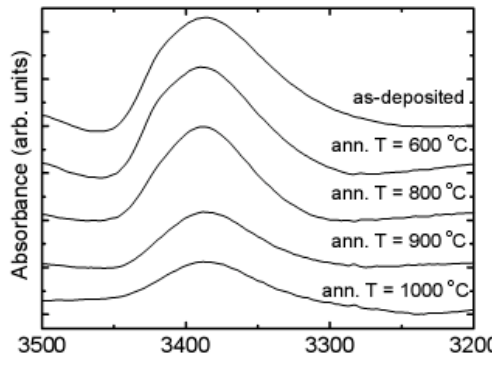

(a)

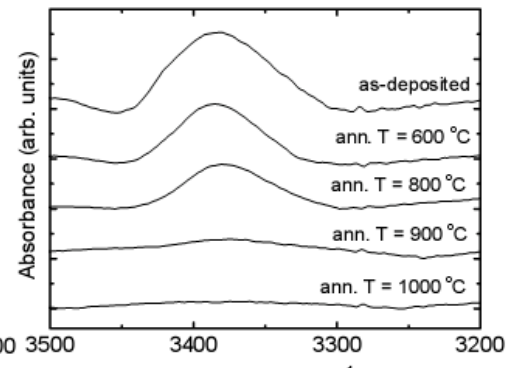

(b) Wavenumber $\left(\mathrm{cm}^{-1}\right)$

Fig. 1 Infrared absorption spectra of (a) undoped and (b) P-doped SiON layers in N-H region under different annealing temperatures (Curves vertically shifted for clarity)

The introduction of B and P also results in obviously improved reflow properties of SiON layer. Fig. 2 shows one example of doped SiON deposited on Si ridges. As can be seen, after reflow the ridges are perfectly covered, which shows the excellent reflow property of the SiON layer. According to our further measurements by atom force microscope (AFM), the root-mean-square surface roughness of the layer after reflow could be lower than one-tenth the value of the as-deposited layer, and a surface roughness as low as $0.16 \mathrm{~nm}$ has been achieved.
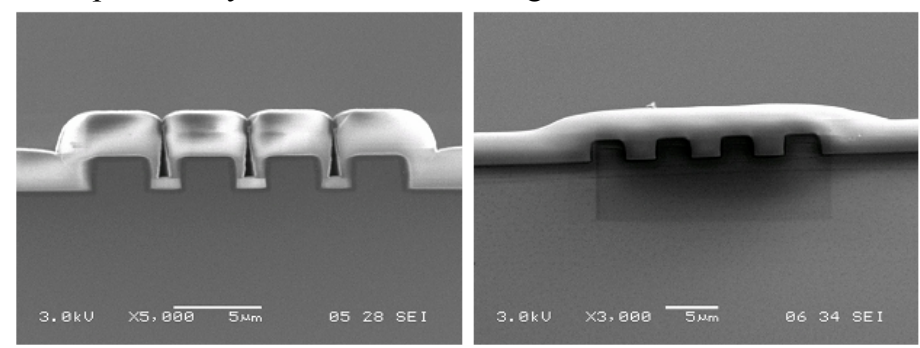

Fig. 2 Cross-section of Si-ridges covered by BP-doped SiON: (a) before and (b) after reflow.

\section{References}

[1] K. Wörhoff, C.G.H. Roeloffzen, R.M. de Ridder, R.M. A. Driessen, and P.V. Lambeck, "Design and Application of Compact and Highly Tolerant Polarization-Independent Waveguides," J. Lightw. Techn. 25, 1276 (2007).

[2] R.M. de Ridder, K. Wörhoff, A. Driessen, P.V. Lambeck and H. Albers, "Silicon Oxynitride Planar Waveguiding Structures for Application in Optical Communication,” IEEE J. Sel. Top. Quant. Electr. 4, 930 (1998).

[3] M.G. Hussein, K. Wörhoff, G. Sengo and A. Driessen, "Optimization of Plasma-enhanced Chemical Vapor Deposition Silicon Oxynitride Layers for Integrated Optics Applications,” Thin Solid Films 515, 3779 (2007).

[4] W.A. Lanford, and M.J. Rand, “The Hydrogen Content of Plasma-deposited Silicon Nitride,” J. Appl. Phys. 49, 2473 (1978). 